\title{
MIR208B Pre-miRNA
}

National Cancer Institute

\section{Source}

National Cancer Institute. MIR208B Pre-miRNA. NCI Thesaurus. Code C82807.

MIR208B is an oligoribonucleotide that is encoded by the human MIR208B gene and has

a role in the regulation of gene expression. 